\title{
Quantitative Model of the Surface Relief Formation in Cyclic Straining
}

\author{
J. POLÁK ${ }^{a, *}$ AND J. MAN ${ }^{b}$ \\ ${ }^{a}$ CEITEC IPM, Institute of Physics of Materials AS CR, Žižkova 22, 61662 Brno, Czech Republic \\ ${ }^{b}$ Institute of Physics of Materials AS CR, Žižkova 22, 61662 Brno, Czech Republic
}

\begin{abstract}
The cyclic strain localization in crystalline materials subjected to cyclic loading results in specific dislocation arrangement in persistent slip bands consisting of alternating dislocation rich and dislocation poor volumes. In the present model the interaction of mobile dislocations in persistent slip band leading to the formation of point defects during cyclic straining is considered. Point defects are steadily produced and simultaneously annihilated due to localized cyclic straining. The non-equilibrium point defects migrate to the matrix and result in transfer of matter between persistent slip band and the matrix and formation of the internal stresses both in the persistent slip band and in the matrix. Plastic relaxation of internal stresses leads to the formation of the characteristic surface relief in the form of persistent slip markings (extrusions and intrusions). Process of point defect migration is quantitatively described and the form of extrusion and parallel intrusions is predicted under some simplifying assumptions. The predictions of the model are discussed and predicted surface relief is compared with selected observations of surface relief produced by cyclic straining.
\end{abstract}

DOI: 10.12693 /APhysPolA.128.675

PACS: 62.20.me, 81.05.Bx, 61.72.Ff, 61.72.Hh, 61.72.Lk, 61.72.J-

\section{Introduction}

Cyclic plastic straining in crystalline materials differs substantially from unidirectional plastic straining in spite of the fact that in both cases dislocation motion plays central role. The dislocation motion in cyclic straining in one direction is severely limited. The repeated unloading and alternating deformation in both directions is typical. Number of stress and strain reversals can achieve several tenths up to several milliards before fracture of the specimen or component takes place. High number of reversal (or cycles) results in very high cumulative strain absorbed by the material during the fatigue life in spite of the fact that the strain introduced in each cycle is very small.

Repeated cyclic plastic straining leads to the formation of specific dislocation structures different from those formed in unidirectional cyclic straining [1]. Moreover, with increasing number of cycles in majority of crystalline materials cyclic plastic strain ceases to be distributed homogeneously and significant inhomogeneities arise. Cyclic plastic strain localization is the substantial feature of the cyclic loading. Inhomogeneous deformation of the material results also in the inhomogeneous distribution of dislocations in the material and dislocation arrangement changes during the fatigue life. The bands of localized slip - persistent slip bands (PSBs) arise. Localized cyclic deformation leads to the formation of the specific surface relief in the form of persistent slip markings (PSMs) which represent the nuclei for fatigue cracks.

\footnotetext{
*corresponding author; e-mail: polak@ipm.cz
}

Dislocation motion in the PSBs has been considered by several authors in the past [2-5]. Number of theories has been proposed to explain the formation of the surface relief and initiation of the early fatigue cracks. Important findings represent experimental data on the resistivity measurements during cyclic plastic straining [6-8] revealing that due to dislocation interactions point defects are steadily produced in cyclic plastic straining. Experimental findings lead to the first attempts to propose physically founded mechanism leading to the formation of the persistent slip markings and later to the initiation of fatigue cracks [4]. Further extension of the model based on the production and migration of point defects in PSBs leads to the explanation of the steady extrusion and intrusion growth [9], to the description of the quantitative growth of extrusions [10] and later to the generalization and quantification of the model of surface relief formation in cyclic plastic straining $[11,12]$.

In the present contribution some experimental data on the dislocation arrangement in the PSBs and on the surface relief produced in cyclic plastic straining are presented. Dislocation motion and interactions within PSBs are considered and production rates of point defects are estimated. The point defect production and migration in the specific dislocation structure of PSBs is quantitatively described and predictions concerning the surface relief are presented.

\section{Dislocation structure of PSBs and characteristic relief of PSMs}

Dislocation arrangement of cyclically deformed materials is studied using transmission electron microscopy (TEM) $[2,3,13,14]$ or using electron channeling contrast (ECC) $[15,16]$. Most studies were performed in copper 
single crystals $[13,14]$ but recently the interest is concentrated to polycrystalline materials [17-19].

Typical dislocation arrangement in fatigued copper single crystal (fcc structure) containing individual PSB is shown in Fig. 1a. Dislocation structure in a grain of fatigued polycrystalline ferritic stainless steel (bcc structure) cycled with low constant plastic strain amplitudes is shown in Fig. 1b. In both cases the foil is perpendicular to the active slip plane and nearly parallel to the primary Burgers vector. PSBs with the ladder-like dislocation structure intersect the basic matrix structure both in copper single crystal and in a grain of ferritic stainless steels. Ladder-like dislocation structure in three dimensions corresponds to alternating thin dislocation-rich walls and thick dislocation-poor channels.
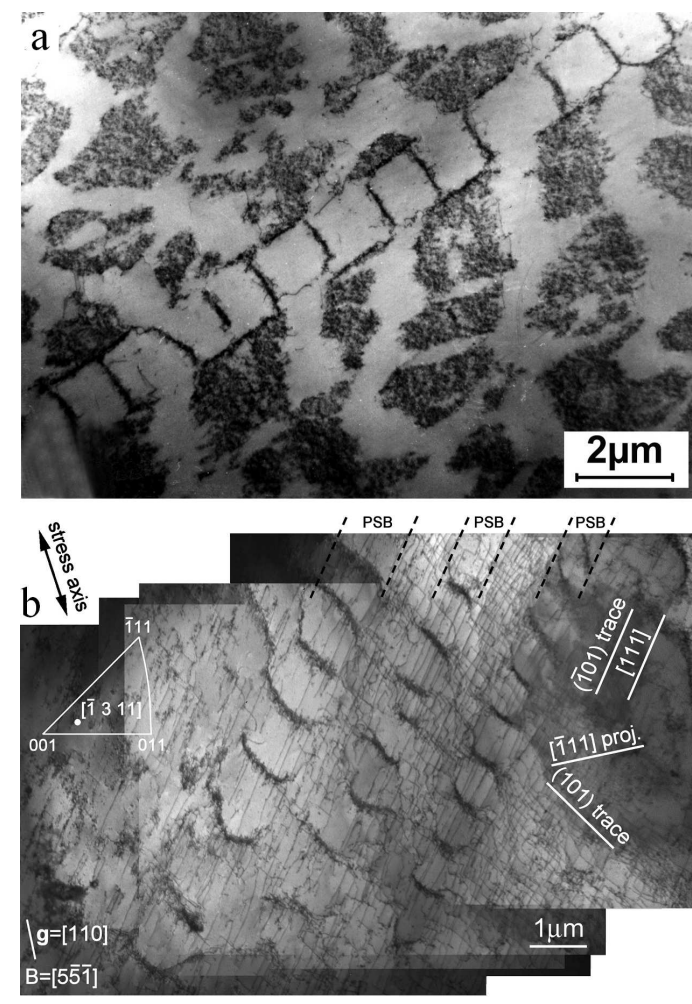

Fig. 1. Dislocation structure in fatigued metals (foils are perpendicular to the to the primary slip plane (a) individual PSB in copper single crystal, (b) parallel PSBs in a grain of ferritic stainless steel.

Surface relief of cyclically deformed materials also witnesses the localized deformation of the material. PSMs arise on the originally smooth surface due to elasticplastic cyclic loading. Figure 2 shows PSMs on the surface of a grain of 316L steel cycled with low plastic strain amplitude. Both the SEM image (Fig. 2a) and AFM image of plastic replica of the surface (Fig. 2b) reveal thin PSMs consisting of extrusions and intrusions and the majority of the surface of the grain is not disturbed by cyclic plastic loading. Plastic replica of the surface was used in order to distinguish the intrusions.

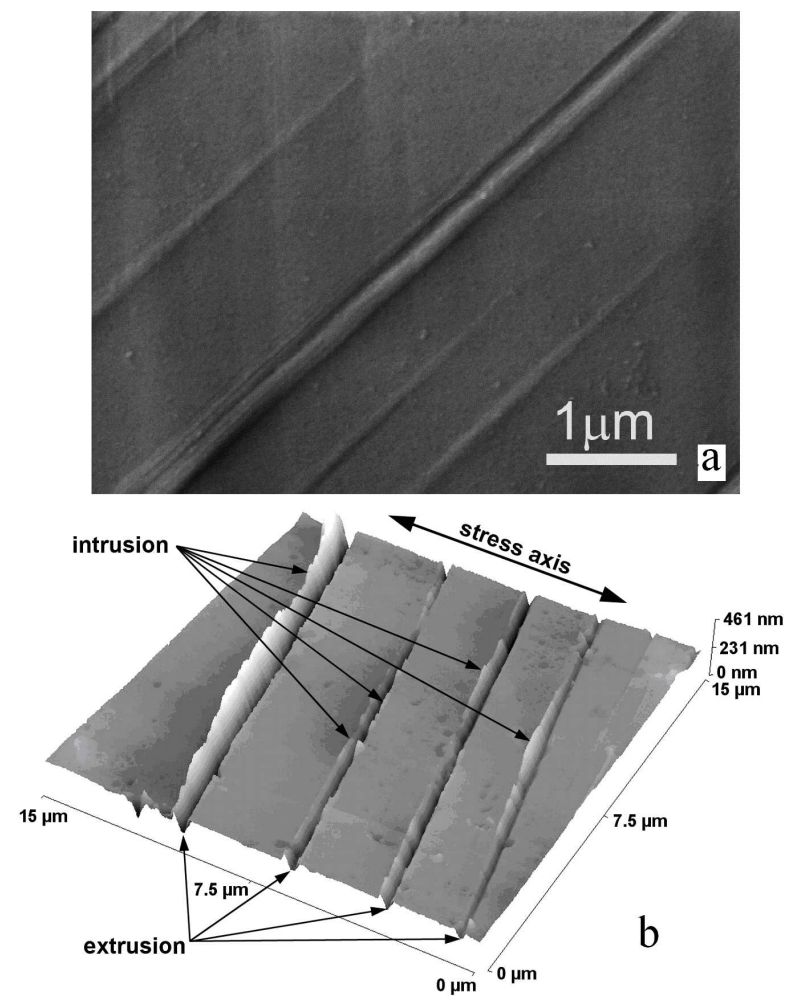

Fig. 2. Surface relief of fatigued 316L steel (a) SEM image of the surface with parallel PSMs, (b) AFM image of the plastic replica of the surface.

\section{Mechanisms of cyclic plastic straining in PSB and point defect production rates}

Basic mechanism of localized cyclic plastic straining in the PSB is shown in Fig. 3. Thin dislocation rich walls consisting mostly of edge dislocation dipoles and multipoles emit dislocation loops in the channels on both sides. Dislocation loops expand and reach the opposite wall, edge segment is built in the wall and most plastic strain is carried by the screw segments. Dislocation arrangement and dislocation interactions within the channel are schematically shown in detail in Fig. 4. Dislocations in

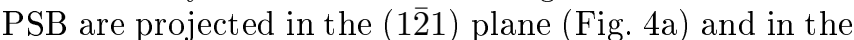
primary (111) plane (Fig. 4b). High plastic strain within a cycle is accommodated by the formation of the dislocation loops starting from the edge dislocation segments in the walls and by their expansion into the channels. The majority of the loops reach the neighbor wall without interaction with other dislocations within the channel and the edge sections become integrated in the dipolar structure of the wall. The largest number of dislocation interactions take place in the walls. Point defects created in the walls are effectively annihilated by short range migration to the edge dislocations in the walls or by mutual interaction of vacancy and interstitial type defects.

Since local plastic strain amplitude within the PSB is high, two dislocation loops expanding from the neighbor walls can interact within a channel. Provided two oppo- 


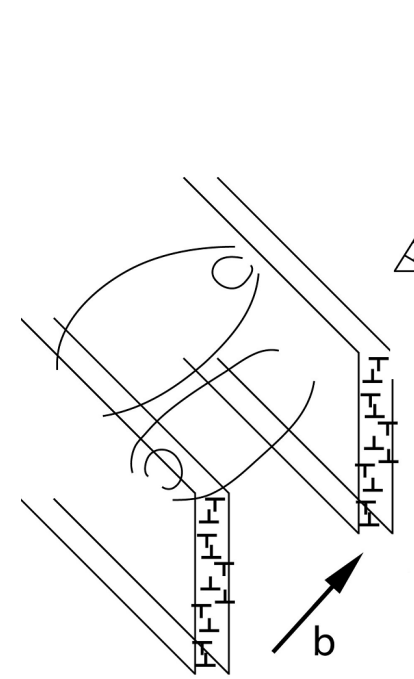

dislocation loops emitted from the walls
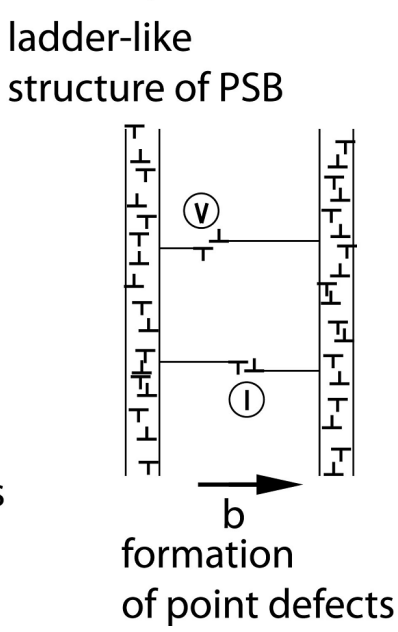

Fig. 3. Schematics of dislocation arrangement and interactions in PSB.

site loops meet on the neighbor planes rows of vacancies and rows of interstitials arise. Polák and Man [11] recently derived production rates of vacancies and interstitials in a PSB subjected to local plastic shear strain amplitude $\gamma_{P}$ (see Fig. 4). Dislocation loops in the PSB are emitted from the walls into the neighbor channels whose width is $d_{c h}$. Some loops can meet within the channel, interact and create the unit dipole corresponding to the row of vacancies or interstitials. The production rate of point defect of type $j$ (vacancy $v$ or interstitial $I$ ) in a cycle $p_{j}$ is

$$
p_{j}=\left(\frac{\mathrm{d} c_{j}}{\mathrm{~d} N}\right)_{p}=2 s_{j} \frac{b}{d_{c h}} \gamma_{p}^{2} .
$$

$s_{j}$ is a proportionality factor, smaller than 1 that is different for unit vacancy dipoles and unit interstitial dipoles. It takes into account whether both dislocations from the opposite walls in a half-cycle are emitted simultaneously. It depends also how far the row of point defects can be extended by the movement of the non-conservative jogs on two screw dislocations formed due to encounter of two loops within a half-cycle period. Since formation energy of the interstitial is much larger than formation energy of a vacancy the length of the interstitial dipole formed during half cycle will be smaller than that of vacancy dipole. Therefore we expect that $p_{I} \ll p_{v}$ and majority of point defect will be vacancy type defects.

Point defects produced during cyclic straining in the channels of the PSB in the form of unit dipoles can be annihilated athermally by encountering an edge dislocation. The slip plane of the edge dislocation must be neighbor plane to the slip plane containing the row of point de-
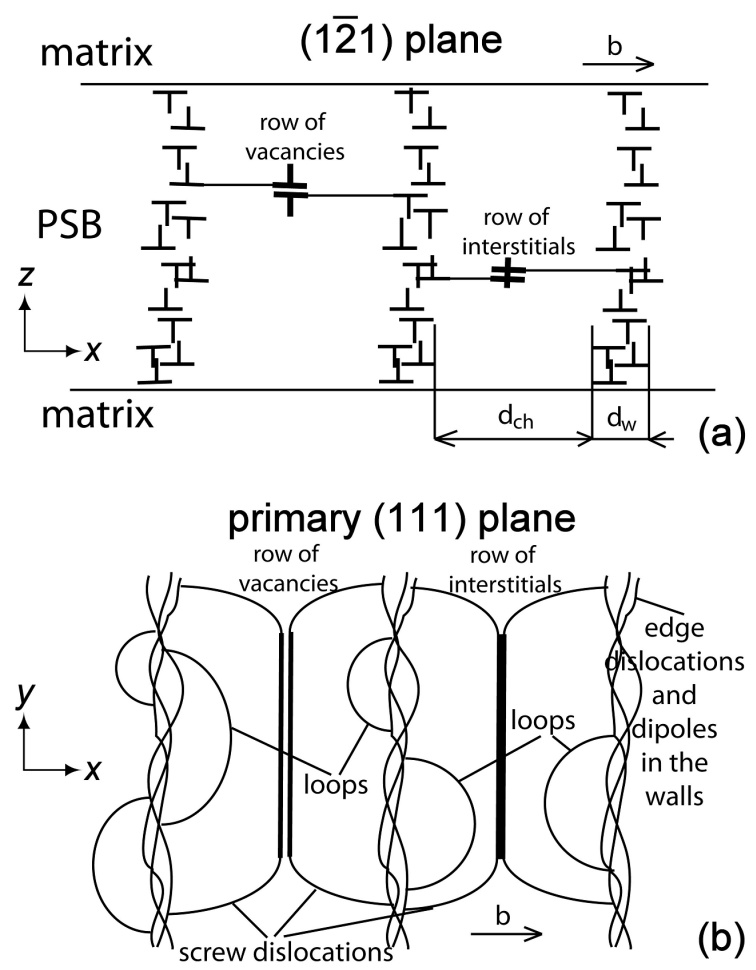

Fig. 4. Schematics of dislocation interactions and formation of point defects in PSB, (a) projection in (12 1$)$ plane, (b) projection in primary (111) slip plane.

fects. On encounter with a row of point defects edge dislocation climbs one atomic distance in appropriate direction. When $c_{j}$ is the concentration of point defect of type $j$ the annihilation rate of a defect of type $j$ (vacancy or interstitial) in a cycle $a_{j}$ was evaluated to [11]:

$$
a_{j}=\left(\frac{\mathrm{d} c_{j}}{\mathrm{~d} N}\right)_{a}=2 \gamma_{p} c_{j} .
$$

The point defect concentration in a PSB with stabilized wall dislocation structure vs. the number of cycles is obtained by equating production and annihilation rates and solving for $c_{j}$ :

$$
c_{j}=s_{j} \frac{b}{d_{c h}} \gamma_{p}\left[1-\exp \left(-2 \gamma_{p} N\right)\right] .
$$

Provided point defects are annihilated only athermally, their concentration increases with increasing number of cycles until saturated value is reached

$$
c_{j, e q}=s_{j} \frac{b}{d_{c h}} \gamma_{p} .
$$

In case point defects do not migrate to sinks the saturated point defect concentration is proportional to the local plastic shear strain amplitude.

\section{Migration of point defects and formation of the surface relief}

Since vacancy production rates are higher, in the following we shall consider only vacancies. They are steadily produced in the whole volume of the PSB with the production rate $p$ (increase of the vacancy concentration in 
a cycle) and simultaneously are annihilated by sweeping dislocations. The width of the PSB is $w$ and its length is $l$ (see schema of PSB in Fig. 5). The annihilation coefficient $A$ is the fraction of vacancies swept by mobile dislocations during one cycle. It was estimated in Sect. 3 to $A=2 \gamma_{p}$. Due to vacancy migration the vacancy concentration $c_{v}$ is a function of the distance $x$ (see Fig. 5). Concentration profile can be obtained by solving appropriate diffusion equations [12]. Migration of vacancies to the walls is neglected (see Sect. 5).

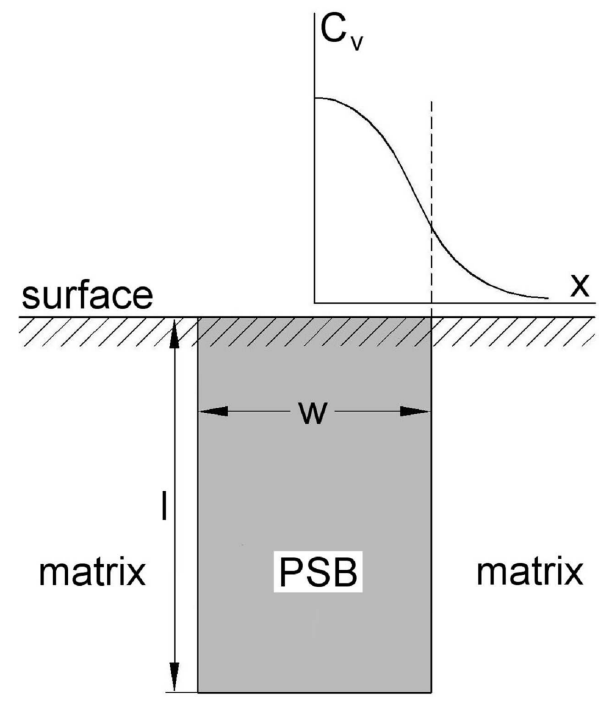

Fig. 5. PSB of the width $w$ and length $l$ embedded in the matrix (artificially inclined perpendicularly to the surface) and the profile of vacancy concentration. Vacancies migrate to the matrix and are annihilated there at edge dislocations.

As soon as the vacancies arrive in the matrix they can be annihilated at edge dislocations. Analytical solution was obtained for homogeneously distributed edge dislocations. Annihilation of vacancies at homogeneously distributed sinks follows first order kinetics and the rate of annihilation is proportional to edge dislocation density $\rho_{e}$ and diffusion coefficient $D_{v}$.

By solving diffusion equation in the PSB and diffusion equation and annihilation at the homogeneously distributed edge dislocations having the density $\rho_{e}$ in the matrix under appropriate boundary conditions the profile of the steady vacancy concentration during cycling was evaluated [12]:

$$
\begin{aligned}
c_{v}= & \frac{p}{A}\left(1-\frac{\cosh (a x)}{\cosh (a w / 2)+\frac{a}{\sqrt{\rho_{e}}} \sinh (a w / 2)}\right) \\
& \text { for } x \leq w / 2
\end{aligned}
$$

and

$$
c_{v}=\frac{p}{A} \frac{\exp \left(-\sqrt{\rho_{e}}(x-w / 2)\right)}{1+\frac{\sqrt{\rho_{e}}}{a} \operatorname{coth}(a w / 2)} \text { for } x \geq w / 2,
$$

where $a=\sqrt{\frac{A}{\tau D_{v}}}$ is the reciprocal characteristic diffusion distance which takes into account vacancy annihilation.
Knowing the steady profile of vacancies in the PSB and in the adjoining matrix we can evaluate the growth rate of the extrusion $(x<w / 2)$ and intrusion $(x>w / 2)$. In the volume element $l d x$ ( $l$ is the length of PSB in the direction of the Burgers vector, see Fig. 5). The rate of the volume change due to migration and annihilation of vacancies is equal to the difference of the vacancy flux in this volume and out of this volume. Vacancy flux $J$ is

$$
J=-D_{v} \frac{\partial c_{v}}{\partial x}
$$

Since absorption of each vacancy corresponds to the arrival (in the PSB) or removal (in the matrix) of one atom from the lattice the internal compression stress (in the PSB) or the internal tensile stress (in the matrix) arise. Three-dimensional internal stresses are added to the external cyclic stress $\sigma$ applied both to PSB and to the matrix. It represents mean stress in the direction of the loading. The mean stress can be relaxed plastically by processes of cyclic creep only in the direction of the Burgers vector of primary dislocations. The relaxation will proceed differently in PSB and in the matrix.

Internal compression stress can be completely relaxed in the PSB by expansion of the material to the surface by process of cyclic creep. The change of the height $h$ of the surface in the direction of the Burgers vector, during one cycle in the distance $x$ from the center of the PSB is thus

$$
\frac{\Delta h}{\Delta N}=-\tau l D_{v} \frac{\mathrm{d}^{2} c_{v}}{\mathrm{~d} x^{2}} .
$$

Inserting Eq. (5) into Eq. (8) and performing the derivative we obtain extrusion growth rate. The extrusion height $h_{E}$ is proportional to the number of loading cycles $N$ :

$$
h_{E}=\frac{p l N \cosh (a x)}{\cosh (a w / 2)+\frac{a}{\sqrt{\rho_{e}}} \sinh (a w / 2)} \text { for } x<w / 2 .
$$

The highest extrusion is produced at the $\mathrm{PSB} /$ matrix interface $(x=w / 2)$.

Since the critical yield stress in the matrix is higher than the critical yield stress in the PSB and the cyclic creep during cycling in the matrix starts only if the component of the mean stress in the direction of the acting cyclic stress reaches the critical value equal to the difference between the yield stress of the matrix and PSB. The intrusion growth will thus start with some delay, i.e. when the critical elastic strain in the particular sheet of the matrix is built up.

At the number of cycles $N$ the depth $d_{I}$ of the intrusion $\left(d_{I}\right.$ is positive $)$ is [12]:

$$
\begin{aligned}
& d_{I}=r_{\operatorname{Im}} N \exp \left(-\sqrt{\rho_{e}}\left(x-\frac{w}{2}\right)\right)-d_{c} \\
& \text { if } r_{\operatorname{Im}} N \exp \left(-\sqrt{\rho_{e}}\left(x-\frac{w}{2}\right)\right)>d_{c} \text { for } x>w / 2,
\end{aligned}
$$

and

$$
\begin{aligned}
d_{I}= & 0 \text { if } r_{\operatorname{Im}} N \exp \left(-\sqrt{\rho_{e}}\left(x-\frac{w}{2}\right)\right) \leq d_{c} \\
& \text { for } x \leq w / 2,
\end{aligned}
$$


where $d_{c}$ is parameter which characterizes the delay between the start of the intrusion growth relative to the onset of the extrusion growth. It is equal to the maximum depth of the hypothetical intrusion (at the $\mathrm{PSB} /$ matrix interface) which would arise provided the yield stress of the PSB and the matrix were the same. $r_{\text {Im }}$ is the maximum intrusion growth rate (at the $\mathrm{PSB} /$ matrix interface) equal to

$$
r_{\operatorname{Im}}=\frac{p l \frac{\sqrt{\rho_{e}}}{a}}{\operatorname{coth}(a w / 2)+\frac{a}{\sqrt{\rho_{e}}}} .
$$

No intrusion will arise until critical number of cycles $N_{c}$ is reached

$$
N_{c}=\frac{d_{c}}{r_{\text {Im }}}
$$

and maximum intrusion depth $d_{\mathrm{Im}}$ at number of cycles $N$ is

$$
d_{\operatorname{Im}}=r_{\operatorname{Im}} N-d_{c} \text {. }
$$

It increases linearly with the number of loading cycles.

Intrusion width $\Delta x_{I}$ is

$$
\Delta x_{I}=\frac{1}{\sqrt{\rho_{e}}} \ln \left(\frac{N r_{\operatorname{Im}}}{d_{c}}\right) .
$$

Figure 6 shows the profile of the PSM (only right part of the symmetrical profile) consisting of the extrusion and intrusion as calculated using Eqs. (9)-(11). The parameters shown in caption to Fig. 6 correspond to cyclically strained copper at room temperature.

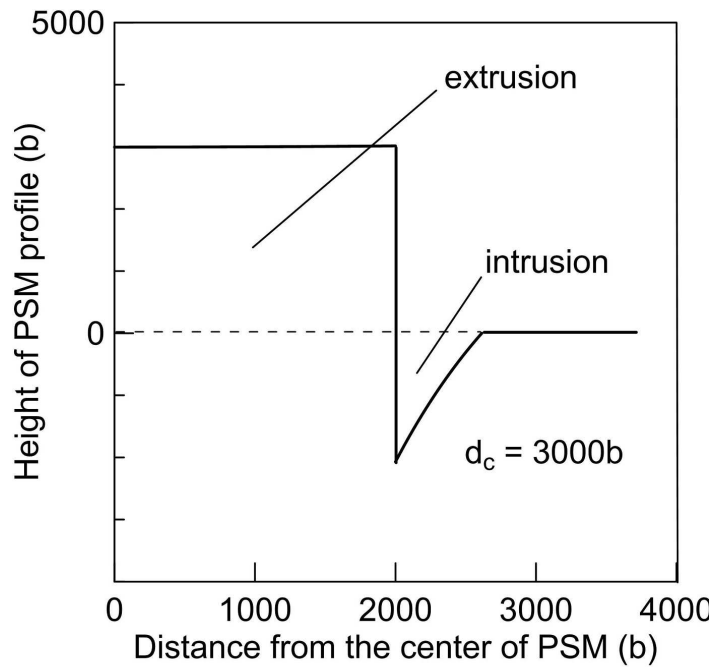

Fig. 6. PSM profile (right part of a symmetrical profile) consisting of extrusion and intrusion calculated using Eqs. (9)-(11). It corresponds to cyclic straining of copper at room temperature for 1000 cycles. The extrusion height in the center $h_{E c}=3000 b$, critical distance is $d_{c}$. Edge dislocation density in the matrix $\rho_{e}=1 \times 10^{-13} \mathrm{~m}^{-2}$. All dimensions are in units of the Burgers vector modulus $b$.

The profile of the extrusion accompanied by two intrusions has been calculated assuming total plastic relaxation of the internal compression and tensile stresses in the PSB and its neighborhood without considering the elastic stresses and strains. In reality, taking into account the elastic strains the transition from extrusion to intrusion will be much smoother than shown in Fig. 6 .

\section{Discussion}

Quantitative relations describing the shape of extrusions and parallel intrusions were derived under several simplifying assumptions. The most limiting assumptions relate to the dislocation arrangement in the PSB and in the neighbor matrix. We have supposed the ladderlike dislocation arrangement of the PSB which allows the majority of produced point defects to escape to the matrix and participate on the production of PSMs. Even in ladder-like structure only fraction of vacancies escapes to the matrix since another fraction is absorbed in dislocation rich walls in the PSB. If the dislocation structure is more irregular as e.g. in 316L steel [18] but still contains the dislocation rich and dislocation poor volumes the fraction of vacancies systematically leaving PSB to the neighbor matrix decreases further. Production rate of vacancies $p$ is reduced and PSM builds up with smaller rate. In Eq. (9) and (10) the production rate $p$ should be replaced by effective production rate $p_{e f f}$.

Much higher effect on the shape of the PSM has the assumption of the homogeneous distribution of edge dislocations in the matrix serving as sinks for vacancies that migrate from the PSB. Some typical distribution of dislocations in the neighborhood of PSBs is illustrated in Fig. 1. In copper (Fig. 1a) the matrix consists of patches and channels with approximately equal sizes. This dislocation structure of the matrix would lead to the wider intrusions since vacancies could migrate larger distances from $\mathrm{PSB} /$ matrix interface before they are annihilated at dislocations. Dislocation structure of the matrix in ferritic stainless steel is close to the assumption of homogeneous distribution. In case the edge dislocation distribution in the matrix close to the PSB/matrix interface is highly inhomogeneous both extrusion height and intrusion depth will vary along the length of the PSM or no intrusion at all will be formed. Variable height of extrusions and depth of intrusions has been often observed [1, 19-21] (see also Fig. 2b).

Comparison of the quantitative predictions of the model with experimental data [19-21] shows reasonable agreement concerning the height of extrusions and intrusions and also their shape. Great achievement of the model is the explanation of the experimental findings that extrusion starts early in the fatigue life and only after significant delay intrusions develop. The model also explains the dependence of the intrusion depth on the local dislocation arrangement in the matrix. In case low edge dislocation density is present in the matrix close to $\mathrm{PSB} /$ matrix boundary the formation of intrusion is delayed significantly or intrusions do not arise at all.

Sharp intrusions grow and become crack-like defect which creates its own cyclic plastic zone. Due to irre- 
versibility of the cyclic plastic strain at the tip of the intrusion it becomes primary stage I fatigue crack. When the growth rate of the stage I crack due to strain irreversibility becomes higher than the growth rate of the intrusion the nucleation of the fatigue crack is accomplished and the crack grows in stage I.

\section{Acknowledgments}

The present work was realized in CEITEC with research infrastructure supported by the project No. CZ.1.05/1.1.00/02.0068. The support by the projects RVO: 68081723 and grants 13-23652S 13-32665S of the Grant Agency of the Czech Republic is gratefully acknowledged.

\section{References}

[1] J. Polák, Cyclic Plasticity and Low Cycle Fatigue Life of Metals, Elsevier, Amsterdam 1991.

[2] J.G. Antonopulos, L.M. Brown, A.T. Winter, Philos. Mag. 34, 549 (1976).

[3] H. Mughrabi, in: Proc. 5th Int. Conf. on the Strength of Metals and Alloys, Eds. P. Hasen, V. Gerold, G. Koster, Vol. 3, Pergamon Press, 1980, p. 1615.

[4] U. Essmann, U. Gösele, H. Mughrabi, Philos. Mag. A 44, 405 (1981).

[5] U. Essmann, Philos. Mag. A 45, 171 (1982).
[6] J. Polák, Czech. J. Phys. B 19, 315 (1969).

[7] J. Polák, Scr. Metall. 4, 761 (1970).

[8] J. Polák, Mater. Sci. Eng. 89, 35 (1987).

[9] J. Polák, Mater. Sci. Eng. 92, 71 (1987).

[10] J. Polák, M. Sauzay, Mater. Sci. Eng. A 500, 122 (2009).

[11] J. Polák, J. Man, Int. J. Fatigue 65, 18 (2014).

[12] J. Polák, J. Man, Mater. Sci. Eng. A 596, 15 (2014).

[13] P. Lukáš, M. Klesnil, J. Krejčí, Phys. Status Solidi 27, 545 (1968).

[14] C. Laird, P. Charsley, H. Mughrabi, Mater. Sci. Eng. 81, 433 (1986).

[15] Y. Kaneko, K. Fukui, S. Hashimoto, Mater. Sci. Eng. A 400-401, 413 (2005).

[16] J. Man, T. Vystavěl, A. Weidner, I. Kuběna, M. Petrenec, T. Kruml, J. Polák, Int. J. Fatigue 39, 44 (2012).

[17] A.T. Winter, Acta Metall. 28, 963 (1980).

[18] K. Obrtlík, T. Kruml, J. Polák, Mater. Sci. Eng. A 187, 1 (1994).

[19] M. Petrenec, J. Polák, K. Obrtlík, J. Man, Acta Mater. 54, 3429 (2006).

[20] J. Man, K. Obrtlik, J. Polák, Philos. Mag. 89, 1295 (2009).

[21] Z.S. Basinski, S.J. Basinski, Prog. Mater. Sci. 36, 89 (1992). 